\title{
How to intervene in the caries process in adults: proximal and secondary caries? An EFCD-ORCA-DGZ expert Delphi consensus statement
}

\author{
Falk Schwendicke ${ }^{1}$ (D) Christian H Splieth ${ }^{2} \cdot$ Peter Bottenberg $^{3} \cdot$ Lorenzo Breschi $^{4} \cdot$ Guglielmo Campus $^{5,6}$. \\ Sophie Doméjean ${ }^{7,8} \cdot$ Kim Ekstrand $^{9} \cdot$ Rodrigo A Giacaman $^{10} \cdot$ Rainer Haak $^{11} \cdot$ Matthias Hannig $^{12} \cdot$ Reinhard Hickel $^{13}$.

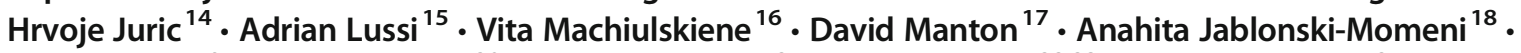 \\ Niek Opdam ${ }^{19}$ - Sebastian Paris ${ }^{20}$ - Ruth Santamaria ${ }^{21} \cdot$ Hervé Tassery $^{22,23}$. Andrea Zandona ${ }^{24}$ - Domenick Zero ${ }^{25}$. \\ Stefan Zimmer ${ }^{26} \cdot$ Avijit Banerjee $^{27}$
}

Received: 23 March 2020 / Accepted: 23 June 2020 / Published online: 9 July 2020

(C) The Author(s) 2020

\begin{abstract}
Objectives To provide consensus recommendations on how to intervene in the caries process in adults, specifically proximal and secondary carious lesions.

Methods Based on two systematic reviews, a consensus conference and followed by an e-Delphi consensus process were held with EFCD/ORCA/DGZ delegates.

Results Managing an individual's caries risk using non-invasive means (oral hygiene measures including flossing/interdental brushes, fluoride application) is recommended, as both proximal and secondary carious lesions may be prevented or their activity reduced. For proximal lesions, only cavitated lesions (confirmed by visual-tactile, or radiographically extending into the middle/ inner dentine third) should be treated invasively/restoratively. Non-cavitated lesions may be successfully arrested using noninvasive measures in low-risk individuals or if radiographically confined to the enamel. In high-risk individuals or if radiographically extended into dentine, for these lesions, additional micro-invasive (lesion sealing and infiltration) treatment should be considered. For restoring proximal lesions, adhesive direct restorations allow minimally invasive, tooth-preserving preparations. Amalgams come with a lower risk of secondary lesions and may be preferable in more clinically complex scenarios, dependent on specific national guidelines. In structurally compromised (especially endodontically treated) teeth, indirect cuspal coverage restorations may be indicated. Detection methods for secondary lesions should be tailored according to the individual's caries risk. Avoiding false positive detection and over-treatment is a priority. Bitewing radiographs should be combined with visualtactile assessment to confirm secondary caries detections. Review/refurbishing/resealing/repairing instead of replacing partially defective restorations should be considered for managing secondary caries, if possible.

Conclusions An individualized and lesion-specific approach is recommended for intervening in the caries process in adults.

Clinical significance Dental clinicians have an increasing number of interventions available for the management of dental caries. Many of them are grounded in the growing understanding of the disease. The best evidence, patients' expectations, clinicians' expertise, and the individual clinical scenario all need to be considered during the decision-making process.
\end{abstract}

Keywords Caries $\cdot$ Consensus $\cdot$ Decision-making $\cdot$ Fluoride $\cdot$ Infiltration $\cdot$ Recommendations $\cdot$ Restorations $\cdot$ Sealing

Falk Schwendicke

falk.schwendicke@charite.de

Extended author information available on the last page of the article

\section{The caries process in adults: proximal and secondary carious lesions}

Much of caries research has been based in the pediatric domain as caries has been regarded classically as predominantly a childhood disease. This was grounded in the past epidemiologic evidence, which did not report significant caries increment in adults. This was due mainly to the fact that restored 
surfaces were not recorded as being able to become "carious" again in these standard epidemiologic assessments. However, as demonstrated by birth cohort studies, the caries increment seems to remain fairly stable from childhood into adult life if accounting for the tooth surfaces at risk $[1,2]$. Caries is therefore as much a disease of adults and older adults as it is of children.

Carious lesions in adults occur primarily on proximal surfaces or are associated with existing restorations (secondary caries) in permanent teeth. The reasons for proximal and secondary caries being a major problem in adults are many.

(1) Occlusal lesions are either successfully prevented longterm by pit-and-fissure sealants [3], or occlusal surfaces are restored during childhood given that active occlusal lesions occur early after the eruption of the tooth and progress relatively fast. In adults, incidence and progression rates of occlusal lesions reduce markedly [4].

(2) Exposed root surfaces are often not evident in this age group. Only later and in conjunction with reduced periodontal support with or without underlying periodontitis [5], root surface carious lesions become more common $[3,10]$.

(3) On proximal surfaces of permanent teeth, lesion development is usually relatively slow. In low caries risk/susceptible patients, several years or even decades may pass until a radiographically detectable lesion occurs [4]. Hence, in children, proximal lesions of permanent teeth are not common as they do not usually have enough time to develop; one exception being the mesial surface of the first permanent molars whilst in contact with the distal surface of the second primary molar which, at the earliest stages, are under risk during a specifically vulnerable time period [6]. Over time and manifesting in adulthood, though, proximal lesions are found developing and progressing frequently, even in well-maintained/motivated populations in highincome countries [7].

(4) Secondary carious lesions are, by definition, associated with existing restorations, with proximally extended restorations showing an increased risk compared with occlusally limited restorations. They occur largely later during adulthood, especially as many younger adults today have fewer restorations which can suffer from secondary lesions [8]. The nature of secondary lesions will be discussed further below, building on the compiled evidence of the systematic reviews underlying this consensus statement.

\section{Materials and methods}

The present consensus statement is part of a series established jointly by the European Federation of Conservative Dentistry (EFCD) and the European Organisation of Caries Research (ORCA). Both organizations strive to promote research and evidence-based practice in the field of cariology and conservative dentistry. Under the tenets of both organizations and following a pilot workshop and consensus process conducted in 2018 on "When to intervene in the caries process?" [9], a consensus process on "How to intervene in the caries process" was initiated in 2019. Due to the high complexity of the question of how to treat dental caries, the topic was divided into three age groups of patients: children and adolescents, adults, and older adults. The present statement specifically deals with the caries process in adults; it focuses on proximal and secondary carious lesions in permanent teeth, two common manifestations of the caries process in this age group. Two other statements focus on caries in children, specifically early childhood caries, primary molar caries and occlusal caries in permanent molars; and older adults, specifically root caries.

The workshop participants had been selected and invited by the boards of ORCA, EFCD, and German Society of Conservative Dentistry (DGZ), achieving a balanced representation of international clinical and academic experts in the disciplines of cariology, restorative dentistry, pediatric dentistry, and gerodontology. Overall, 25 delegates participated in the process. The costs of the workshop were covered solely by the participating dental associations. To summarize the current scientific knowledge, selected members of the three associations had been asked before to prepare systematic literature reviews regarding the treatment of most prevalent caries forms in the three age groups. The reviews were published in Caries Research and Clinical Oral Investigations.

Based on these reviews as well as other literature from structured consensus statements, recommendations were drafted by the group chairs (FS, AB) and sent to the delegates prior to the meeting. At the meeting, each statement was discussed extensively and modified until consensus was reached. The strength of each recommendation was evaluated by the group as being "strong," "moderate," or "weak," based on the scientific evidence supporting the statement. Recommendations supported by unequivocal evidence (e.g., several high-quality randomized controlled trials) were as evaluated as "strong." Recommendations based on moderate evidence (e.g., high-quality clinical studies with similar outcomes) were evaluated as "moderate." Finally, recommendations based on expert opinion only or on low-quality studies or studies with contradicting outcomes were evaluated as "weak."

The voting on the statement was graded from 1 (completely disagree) to 10 (completely agree) and performed using an online platform (Surveyjet, Calibrum, St. George, USA). At least $70 \%$ of the votes over 7 were considered as acceptance of the statement by the group, and in addition, the median of all votes was calculated. An additional field for free-text comments was also available to illustrate the reasoning for a certain decision or proposal for future modifications.

The aim of the present statement is to compile the best available evidence, appraise it, and use it to derive clinically applicable, consented recommendations on how to intervene 
in the caries process in adults. It is specifically developed to support decision-making in pragmatic clinical practice and while all recommendations are consented (see below); their scientific evidence base is often limited, as indicated. Hence, this statement also highlights research gaps for potential future investigation. Before presenting the findings of the systematic reviews and the resulting recommendations, a brief overview of caries management in adults including some detail with respect to the types of carious lesions occurring more commonly in the adult age group will be discussed.

\section{Results}

\section{How to intervene in proximal lesions}

A recent consensus statement [9] established guidelines on when to intervene on proximal lesions. It was agreed that for non-cavitated lesions, minimally invasive (restorative) strategies should be avoided, and non- or micro-invasive strategies are preferable. Non-invasive strategies do not remove dental hard tissue and involve, for example, topical fluorides and other chemical agents for controlling mineral balance, biofilm control measures, and dietary control. Micro-invasive strategies remove the dental hard tissue surface at the micron level, usually during an etching step, such as used in sealing or infiltration techniques. Minimally invasive operative strategies remove a limited amount of gross dental hard tissue, through the use of hand excavators, rotary instruments, or other devices. In most cases, this process is associated with the subsequent placement of restorations. The specific types of non- and micro-invasive strategies have been laid out in more detail in the systematic review underlying this consensus statement, together with the evidence supporting them (see Table 1 and below). Cavitation status should be assessed using visual and/or tactile methods, and/or the radiographic lesion depth as a proxy, with cavitation being unlikely in lesions radiographically confined to enamel, likely in those clearly entering the middle third of dentine, and variable for lesions around the enamel-dentine junction/outer third of the dentine.

The systematic review [10] identified mainly studies combining oral hygiene measures and fluoride application for managing proximal lesions, either as part of long-term cohort studies or as control arm of randomized trials comparing nonand micro-invasive strategies for managing proximal lesions. Overall, in most low caries risk/susceptible populations, lesion progression was slow, taking several years. Progression was more rapid in lesions extending radiographically into dentine than in enamel alone, in molars rather than premolars, and in adolescents rather than adults. Notably, in highly caries susceptible individuals, lesion progression can be faster, with non-cavitated lesions progressing to cavitated dentine lesions over a 1-2-year period. Generally, non-invasive measures depend on the potential for patients' behavioral modification and adherence which is not often considered in such studies [57]. Overall, the evidence supporting non-invasive management of proximal lesions as a therapy vs. doing nothing was graded weak. However, as non-invasive measures also aim to modify a patient's caries risk/susceptibility, they are relevant clinically as part of fundamental disease prevention.

For micro-invasive management of proximal lesions, a larger and more robust body of evidence was available, with a number of randomized trials assessing micro-invasive treatment combined with non-invasive measures vs. non-invasive management alone. The studies found that micro-invasive treatment combined with non-invasive measures arrests noncavitated enamel and initial dentine lesions (limited to the outer third of dentine based on radiograph and clinically non-cavitated) and is significantly more effective than noninvasive management alone. However, it remained unclear which micro-invasive technique offered the greatest benefit. There was some evidence that both sealing and resin infiltration can arrest enamel-limited lesions and those around the enamel-dentine junction, while only infiltration techniques were effective for lesions involving the dentine. Overall, the evidence supporting micro-invasive treatment combined with non-invasive measures of proximal lesions was graded as moderate.

For the minimally invasive restorative/operative management of proximal lesions, a larger number of studies were available. Mean annual failure rates varied between 1.2 and $3.8 \%$ (after weighting for sample size). When restricting to only proximal restorations, conventional resin composites showed a significantly higher risk of failure than amalgams; all other materials did not perform significantly differently than conventional resin composites. Notably, though, studies were conducted at different times, with different materials and different operator experience, which makes comparisons difficult. Overall, the evidence was graded as weak. Amalgams appear to show a lower risk of failure in proximal cavities than most other materials. In light of many patients wishing to receive tooth-colored restorations, but also because the aim of minimally invasive tooth tissue preservation, by biologically oriented lesion preparation, as well as considering the current global phase-down of dental amalgam associated with the Minamata agreement [11], adhesive bio-interactive restorations are common in many countries. For extensive defects, especially in endodontically treated teeth, indirect restorations may also be considered on an individualized basis.

Based on these findings for proximal lesions, the consensus group developed the following recommendations:

1. Overall, the management of proximal lesions should be provided under the tenet of a preventively oriented approach (dietary advice, oral hygiene motivation). Managing a patient's caries risk/susceptibility will also 
Table 1 Non- and micro-invasive strategies for managing proximal lesions and the associated evidence base

\begin{tabular}{|c|c|}
\hline Strategies & Evidence strength \\
\hline \multicolumn{2}{|l|}{ Non-invasive } \\
\hline $\begin{array}{l}\text { Oral hygiene measures, mainly flossing and } \\
\text { interdental brushes }\end{array}$ & $\begin{array}{l}\text { Weak. Randomized studies find these measures to remove } \\
\text { plaque and prevent gingivitis, but not necessarily prevent } \\
\text { or manage existing caries. }\end{array}$ \\
\hline Fluoride (varnish, gel) application & $\begin{array}{l}\text { Weak. No studies available testing this approach only; } \\
\text { most combined it with oral hygiene. }\end{array}$ \\
\hline Combined oral hygiene and fluoride & $\begin{array}{l}\text { Weak. Both randomized studies and cohort studies available } \\
\text { using combinations of oral hygiene and fluoride. However, } \\
\text { fluoride application likely more relevant (see above). } \\
\text { Proximal lesion arrest or slowing down lesion progression } \\
\text { seems viable using this measure. }\end{array}$ \\
\hline Dietary advice & $\begin{array}{l}\text { Weak. No specific data supporting this measure for } \\
\text { managing proximal lesions. Biologic plausibility is, } \\
\text { however, given. }\end{array}$ \\
\hline \multicolumn{2}{|l|}{ Micro-invasive } \\
\hline $\begin{array}{l}\text { Caries sealing (lesion is conditioned using phosphoric } \\
\text { acid and then sealed using an adhesive or flowable } \\
\text { composite, or glass ionomer cement; mechanism } \\
\text { of "diffusion barrier" installation) }\end{array}$ & $\begin{array}{l}\text { Weak. Randomized trials support caries sealing } \\
\text { (mainly using resins) in addition to non-invasive measures } \\
\text { for early lesions, with the majority being arrested. Applicability } \\
\text { in full dental arch unclear, though. }\end{array}$ \\
\hline $\begin{array}{l}\text { Caries infiltration (lesion is conditioned using } \\
\text { hydrochloric acid, dried, and a low-viscosity resin } \\
\text { allowed to penetrate into the lesion body. After } \\
\text { light-curing, a "diffusion barrier" has been established) }\end{array}$ & $\begin{array}{l}\text { Moderate. Randomized trials support caries infiltration in addition } \\
\text { to non-invasive measures, with nearly all lesions being arrested. } \\
\text { Applicability given also in full dental arch. Currently only } \\
\text { one infiltration system on the market. }\end{array}$ \\
\hline
\end{tabular}

help support managing specific proximal lesions and assist in the prevention of new lesions on unaffected surfaces.

2. For non-cavitated lesions:

a. Non-invasive measures (e.g., interdental cleaning, topical fluoride application) could be applied to arrest proximal lesions. This may be sufficient for lesion arrest in low caries risk/susceptible individuals or for lesions radiographically confined to enamel (weak recommendation, agreement $88 \%$, median: 10 )

b. In high-risk/susceptible individuals or for lesions extending radiographically into dentine, micro-invasive strategies should be considered additionally (moderate recommendation, agreement $83 \%$, median: 10 ).

c. The decision between sealing and resin infiltration should be guided by individual considerations, including applicability, clinical experience, or costs (moderate recommendation, agreement $88 \%$, median: $10)$.

3. For cavitated lesions, restorative strategies will often be needed. For restoring proximal lesions, adhesive direct restorations allow minimally invasive, tooth-preserving preparations, are tooth-colored, and hence are already the material of choice in many cases. Amalgams, however, come with a lower risk of secondary lesions and since their placement is less technique-sensitive, they may be preferred in more clinically complex scenarios, dependent on specific national guidelines. According to legal regulations, the use of amalgam may be restricted in some countries or populations, now and in the future (weak recommendation, agreement $84 \%$, median: 10 ).

4. In structurally compromised teeth, especially when endodontically treated, indirect cuspal coverage restorations may be indicated (weak recommendation, agreement 92\%, median: 10).

\section{How to intervene in secondary lesions}

Secondary lesions may be primary carious lesions associated with existing restorations, as a result of the caries process not having been sufficiently addressed on a patient level [12-14], or may be caused by significant margin defects (interfacial gaps, ditches, etc.), allowing acidic by-products or a dysbiotic biofilm to enter the interface between the restoration and the tooth structure. Marginal gaps may be the result of imperfect initial placement of the restoration/management of the tooth surface [15], but also hydrolytic degradation of the hybrid layer of adhesive restorations $[16,17]$. In any case, secondary lesions are found more often in high caries risk/susceptible patients [18, 19], at the gingival margin of restorations [20, $21]$, and in posterior teeth [22]. 
The second review underlying this consensus statement assessed a number of aspects related to secondary carious lesions:

(1) Can secondary lesions be managed using specific restorative techniques or materials?

(2) How can secondary lesions be detected, allowing their adequate management?

(3) If/when detected, which management strategy can be recommended?

Regarding the risk of secondary carious lesions next to restorations placed using different adhesive strategies and restorative materials, the review yielded only a few robust findings [23]. Overall, amalgams appear to be associated with a lower risk of secondary caries [24], which is in agreement with amalgams showing an overall lower risk of failure in most cavities (see above). Again, however, many dentists will be interested in knowing which non-amalgam materials are least prone to secondary lesions. For these materials (different resin composite types, but also resin-modified or conventional glass-ionomer cement or poly-acid modified resin composites/ compomers), only very few differences emerged. Overall, patient- and operator-level factors seem to be decisive in controlling secondary carious lesions, while adhesive strategies and/or restorative materials may play a lesser role. This conclusion, however, is limited by the short follow-up periods, limited sample sizes, and the potential of low caries risk/susceptibility individuals enrolled in most trials.

Early detection of secondary lesions may allow the application of less invasive treatment options like surface refurbishment, re-sealing, or repair instead of complete restoration removal and replacement. A variety of early detection methods for secondary carious lesions are available, including visual, tactile, radiographic, laser fluorescence, and quantitative light-induced fluorescence assessments. These were assessed in a recent review [25], which found that visual, radiographic, and laser fluorescence detection had similar sensitivities and specificities (abilities to detect carious and sound surfaces, respectively), while tactile assessment and light-induced fluorescence are not as useful at present, at least not if used in isolation. There is further data demonstrating that in most low caries risk populations, avoiding false-positive readings and ultimate over-detection and treatment should be prioritized, as secondary lesions progress slowly (see above) and missed lesions may be detected at the next recall consultation [26]. Overall, it seems advisable to combine bitewing radiography (allowing the detection of clinically inaccessible lesions) with visual-tactile assessment (allowing confirmation of findings). If detected as suspect, but not treated, lesions should be monitored regularly. Moreover, dentists should consider to refurbish, reseal, or repair partially defective restorations if possible, as this allows the preservation of tooth structure and reduces the risk of subsequent treatment-related complications [27]. The evidence quality supporting the decision between refurbishment/reseal/repair vs. replacement, however, was found to be weak.
Despite the obvious advantages of this minimally invasive approach (reduced tissue removal, reduced harm/pulp damage, lifetime of existing tooth-restoration complex is prolonged), there may be some other factors to consider (possibly higher long-term costs, careful case selection is required). Overall, the decision will need to consider the specific clinical situation (is repair even possible?), the patient's wishes, and the dentist's experience and expertise with different treatments.

Based on these findings for secondary carious lesions, the consensus group developed the following recommendations:

1. By managing a patient's overall caries risk/susceptibility, the risk for secondary lesion occurrence is also managed to a certain extent (weak recommendation, agreement 87\%, median: 10 ).

2. Detection methods for secondary lesions should be tailored according to patients' caries risk/susceptibility. Especially in low-risk patients, false-positive detection, and subsequent over-treatment should be avoided. This may be achieved by combining bitewing radiography and visual-tactile assessment/confirmation when screening for secondary lesions (weak recommendation, agreement $88 \%$, median: 10 ).

3. When managing detected secondary lesions, dentists should adopt the minimally invasive approaches of refurbishment/reseal/repair over replacement of restorations, on a case-by-case basis (weak recommendation, agreement $100 \%$, median: 10 ).

\section{Conclusions}

The management of carious lesions and caries experience is a daily routine for dentists worldwide. In adults, two specific lesion types are managed frequently: proximal and secondary lesions. The present consensus statement provides agreed recommendations for managing these lesions. Dental clinicians should consider these recommendations in their daily decision-making and apply them depending on the patients' wishes, the individual clinical needs, and their experience and expertise.

Funding statement This study was supported by EFCD, ORCA, and DGZ.

Author contributions The study was conceived by FS, CHS, SP, and AB. FS and $\mathrm{AB}$ wrote the manuscript. All authors read and approved the manuscript.

Funding Information Open Access funding provided by Projekt DEAL.

\section{Compliance with ethical standards}

Competing interests $\mathrm{AB}$ received support for a researcher-led SR of sugar-free gum by Mars Wrigley. PB received an honorarium for 
consultancy on product development regarding future caries treatments in medically compromised adults from Mayser Pharma. LB received support for research protocols regarding dental adhesives from $3 \mathrm{M}$ ESPE, Ivoclar Vivadent, Dentsply Sirona, VOCO, Heraeus Kulzer, Kuraray Noritake, Ultradent, and Pulpdent and material samples for this research from 3 M ESPE, Ivoclar Vivadent, Dentsply Sirona, VOCO, Heraeus Kulzer, Kuraray Noritake, Ultradent, Sweden\&Martina, DMG, and Pulpdent. RAG received dental materials from DMG and from $3 \mathrm{M}$ Oral Care to support some of the research cited in this paper. DJM received support for conference lecturing and attendance by dental manufacturing companies DMG, GC Corporation, and SDI. NO received financial support for a practice-based research network from Kuraray Europe. SP received royalties (Charité-hold patents on caries infiltration) from DMG and honorarium from GSK, CP GABA, and DMG. FS received an honorarium for consultancy on product development regarding future caries treatments, study support on radiopaque tagging of caries lesions, restoring teeth with selective caries excavation, for organizing IADR Symposia on caries treatment from DMG. CHS received research and travel support from SDI regarding the use of silver diammine fluoride. AFZ received an honorarium for consultancy on product development regarding future caries treatments from Colgate, GreenMark, and Calcivis; research support from Colgate, NIDCR, Greenmark, Delta Dental, and Calcivis; non-monetary support (e.g., equipment, facilities, research assistants, paid travel to meetings) for an IADR Symposia on caries detection by Calcivis. DZ received an honorarium for consultancy from Colgate Palmolive, Procter \& Gamble, Greenmark Biomedical Inc., GlaxoSmithKline, and Johnson \& Johnson, and research support from Novatis Pharmaceuticals, Johnson \& Johnson, Univerlever, Hello Products, and Church and Dwight.

Open Access This article is licensed under a Creative Commons Attribution 4.0 International License, which permits use, sharing, adaptation, distribution and reproduction in any medium or format, as long as you give appropriate credit to the original author(s) and the source, provide a link to the Creative Commons licence, and indicate if changes were made. The images or other third party material in this article are included in the article's Creative Commons licence, unless indicated otherwise in a credit line to the material. If material is not included in the article's Creative Commons licence and your intended use is not permitted by statutory regulation or exceeds the permitted use, you will need to obtain permission directly from the copyright holder. To view a copy of this licence, visit http://creativecommons.org/licenses/by/4.0/.

\section{References}

1. Broadbent JM, Foster Page LA, Thomson WM, Poulton R (2013) Permanent dentition caries through the first half of life. Br Dent J 215(7):E12

2. Broadbent JM, Thomson WM, Poulton R (2008) Trajectory patterns of dental caries experience in the permanent dentition to the fourth decade of life. J Dent Res 87(1):69-72

3. A. Ahovuo-Saloranta, H. Forss, T. Walsh, A. Nordblad, M. Makela, H.V. Worthington(2017), Pit and fissure sealants for preventing dental decay in permanent teeth, Cochrane Database Syst Rev 7 Cd001830

4. Mejàre I, Stenlund H, Zelezny-Holmlund C (2004) Caries incidence and lesion progression from adolescence to young adulthood: a prospective 15-year cohort study in Sweden. Caries Res 38(2):130-141

5. Papapanou PN, Sanz M, Buduneli N, Dietrich T, Feres M, Daniel HF, Thomas FF, Garcia R, William VG, Graziani F, Greenwell H, Herrera D, Richard TK, Kebschull M, Denis FK, Keith LK, Kocher T, Kenneth SK, Purnima SK, Bruno GL, Machtei E, Meng H, Mombelli A, Needleman I, Offenbacher S, Gregory JS, Teles R, Maurizio ST (2018) Periodontitis: consensus report of workgroup 2 of the 2017 World Workshop on the Classification of Periodontal and Peri-Implant Diseases and Conditions. Journal of Clinical Periodontology 45(S20):S162-S170

6. Mejàre I, Stenlund H, Julihn A, Larsson I, Permert L (2001) Influence of approximal caries in primary molars on caries rate for the mesial surface of the first permanent molar in Swedish children from 6 to 12 years of age. Caries Res 35(3):178-185

7. Mejàre I, Källestål C, Stenlund H (1999) Incidence and progression of approximal caries from 11 to 22 years of age in Sweden: a prospective radiographic study. Caries Res 33(2):93-100

8. Jordan RA, Krois J, Schiffner U, Micheelis W, Schwendicke F (2019) Trends in caries experience in the permanent dentition in Germany 1997-2014, and projection to 2030: morbidity shifts in an aging society. Sci Rep 9(1):5534

9. Schwendicke F, Splieth C, Breschi L, Banerjee A, Fontana M, Paris S, Burrow MF, Crombie F, Page LF, Gaton-Hernandez P, Giacaman R, Gugnani N, Hickel R, Jordan RA, Leal S, Lo E, Tassery H, Thomson WM, Manton DJ (2019) When to intervene in the caries process? An expert Delphi consensus statement, Clin Oral Investig

10. Splieth CH, Kanzow P, Wiegand A, Schmoeckel J, JablonskiMomeni A (2020) How to intervene in the caries process: proximal caries in adolescents and adults-a systematic review and meta-analysis. Clin Oral Investig 24(5):1623-1636

11. United Nations Environmental Programme (2013) Minamata convention on mercury. United Nations, New York

12. Kidd EA (2001) Diagnosis of secondary caries. J Dent Educ 65(10): 997-1000

13. Schwendicke F, Kern M, Blunck U, Dorfer C, Drenck J, Paris S (2014) Marginal integrity and secondary caries of selectively excavated teeth in vitro. J Dent 42(10):1261-1268

14. Ferracane JL (2017) Models of caries formation around dental composite restorations. J Dent Res 96(4):364-371

15. Vandewalle KS, Ferracane JL, Hilton TJ, Erickson RL, Sakaguchi RL (2004) Effect of energy density on properties and marginal integrity of posterior resin composite restorations. Dent Mater 20(1):96-106

16. Liu Y, Tjaderhane L, Breschi L, Mazzoni A, Li N, Mao J, Pashley DH, Tay FR (2011) Limitations in bonding to dentin and experimental strategies to prevent bond degradation. J Dent Res 90(8):953-968

17. Tjaderhane L (2015) Dentin bonding: can we make it last? Oper Dent 40(1):4-18

18. Opdam NJ, Bronkhorst EM, Roeters JM, Loomans BA (2007) A retrospective clinical study on longevity of posterior composite and amalgam restorations. Dent Mater 23(1):2-8

19. van de Sande FH, Opdam NJ, Rodolpho PA, Correa MB, Demarco FF, Cenci MS (2013) Patient risk factors' influence on survival of posterior composites. J Dent Res 92(7 Suppl):78s-83s

20. Mjor IA (1985) Frequency of secondary caries at various anatomical locations. Oper Dent 10(3):88-92

21. Mjor IA (2005) Clinical diagnosis of recurrent caries. J Am Dent Assoc 136(10):1426-1433

22. Demarco FF, Collares K, Coelho-de-Souza FH, Correa MB, Cenci MS, Moraes RR, Opdam NJM (2015) Anterior composite restorations: a systematic review on long-term survival and reasons for failure. Dent Mater 31(10):1214-1224

23. Askar H, Krois J, Göstemeyer G, Bottenberg P, Zero D, Banerjee A, Schwendicke F (2020) Secondary caries: what is it, and how it can be controlled, detected, and managed? Clin Oral Investig 24(5):1869-1876

24. Moraschini V, Fai CK, Alto RM, Dos Santos GO (2015) Amalgam and resin composite longevity of posterior restorations: a systematic review and meta-analysis. J Dent 43(9):1043-1050

25. Brouwer F, Askar H, Paris S, Schwendicke F (2016) Detecting secondary caries lesions: a systematic review and meta-analysis. J Dent Res 95(2):143-151 
26. Schwendicke F, Brouwer F, Paris S, Stolpe M (2016) Detecting proximal secondary caries lesions: a cost-effectiveness analysis. J Dent Res 95(2):152-159

27. D. Green, L. Mackenzie, A. Banerjee, Minimally invasive longterm management of direct restorations: the ' 5 Rs', Dent Update $42(5)(2015)$ 413-6, 419-21, 423-6
Publisher's note Springer Nature remains neutral with regard to jurisdictional claims in published maps and institutional affiliations.

\section{Affiliations}

Falk Schwendicke ${ }^{1}$ (1) - Christian H Splieth ${ }^{2}$ - Peter Bottenberg ${ }^{3} \cdot$ Lorenzo Breschi $^{4}$ - Guglielmo Campus ${ }^{5,6}$. Sophie Doméjean ${ }^{7,8} \cdot$ Kim Ekstrand $^{9} \cdot$ Rodrigo A Giacaman $^{10} \cdot$ Rainer Haak $^{11} \cdot$ Matthias Hannig $^{12} \cdot$ Reinhard Hickel $^{13}$. Hrvoje Juric ${ }^{14}$. Adrian Lussi ${ }^{15} \cdot$ Vita Machiulskiene ${ }^{16}$. David Manton ${ }^{17}$. Anahita Jablonski-Momeni ${ }^{18} \cdot$ Niek Opdam $^{19}$. Sebastian Paris ${ }^{20} \cdot$ Ruth Santamaria $^{21} \cdot$ Hervé Tassery ${ }^{22,23} \cdot$ Andrea Zandona $^{24} \cdot$ Domenick Zero $^{25} \cdot$ Stefan Zimmer $^{26}$. Avijit Banerjee ${ }^{27}$

1 Department of Operative Dentistry, Charité - Universitätsmedizin, Berlin, Germany

2 Preventive and Pediatric Dentistry, Center for Oral Health, Universitätsmedizin Greifswald, Greifswald, Germany

3 Oral Health Research Group, Vrije Universiteit Brussel, Brussel, Belgium

4 Department of Biomedical and Neuromotor Sciences, DIBINEM, University of Bologna - Alma Mater Studiorum, Bologna, Italy

5 Department of Restorative, Preventive and Paediatric Dentistry, Zahnmedizinische Kliniken (ZMK), University of Bern, Freiburgstrasse 7, 3010 Bern, Switzerland

6 Department of Surgery, Microsurgery and Medicine Sciences, School of Dentistry, University of Sassari, Sassari, Italy

7 Département d'Odontologie Conservatrice, Univ Clermont Auvergne, UFR d'Odontologie; Centre de Recherche en Odontologie Clinique EA 4847, F-63100 Clermont-Ferrand, France

8 CHU Estaing Clermont-Ferrand, Service d'Odontologie, F63001 Clermont-Ferrand, France

9 Cariology and Endodontics, Department of Odontology, Faculty of Health and Medical Sciences, University of Copenhagen, Copenhagen, Denmark

10 Cariology Unit, Department of Oral Rehabilitation, Faculty of Health Sciences, University of Talca, Talca, Chile

11 Department of Cariology, Endodontology and Periodontology, University Leipzig, Leipzig, Germany

12 Clinic of Operative Dentistry and Periodontology, Saarland University, Universitätsklinikum des Saarlandes, Homburg/ Saar, Germany

13 Department of Conservative Dentistry and Periodontology, University Hospital, LMU Munich, Munich, Germany

14 Department of Paediatric and Preventive Dentistry, School of Dental Medicine, University of Zagreb, Zagreb, Croatia
15 School of Dental Medicine, University of Bern, Bern, Switzerland and Department of Operative Dentistry and Periodontology, Faculty of Dentistry, University Medical Centre, Freiburg, Germany

16 Clinic of Dental and Oral Pathology, Faculty of odontology, Lithuanian University of Health Sciences, Kaunas, Lithuania

17 Centrum van Tandheelkunde en Mondzorgkunde, UMCG, Groningen, Netherlands

18 Philipps-University Marburg, Dental School, Department of Orthodontics, Marburg, Germany

19 Radboud University Medical Centre, Department of Dentistry, Radboud Institute for Health Sciences, Nijmegen, The Netherlands

20 Department of Operative Dentistry, Charité - Universitätsmedizin Berlin, Berlin, Germany

21 Preventive and Pediatric Dentistry, Center for Oral Health, Universitätsmedizin Greifswald, Greifswald, Germany

22 Faculté d'Odontologie Marseille, Preventive and Restorative Department, Marseille cedex, Aix-Marseille-Université, Marseille, France

23 EA 4203 Laboratory, Université de Montpellier, Montpellier, France

24 Department of Comprehensive Care, School of Dental Medicine, Tufts University, Boston, Massachusetts, USA

25 Department of Cariology, Operative Dentistry and Dental Public Health, Oral Health Research Institute, School of Dentistry Indiana University, Indianapolis, IN, USA

26 Department of Operative and Preventive Dentistry, Faculty of Health, Dental School, Witten/Herdecke University, Witten, Germany

27 Conservative \& MI Dentistry, Faculty of Dentistry, Oral \& Craniofacial Sciences, King's College London, London, UK 